\title{
BMJ Open Quality Oxygen prescription: improving compliance using methods from BMJ Open Quality journal
}

\author{
Onn Shaun Thein, ${ }^{1,2}$ Cathleen Man Ting Chan, ${ }^{3}$ Eleanor McCance, ${ }^{4}$ Maria Mullins, ${ }^{4}$ \\ Davinder Dosanjh ${ }^{2}$
}

To cite: Thein OS, Chan CMT, McCance $\mathrm{E}$, et al. Oxygen prescription: improving compliance using methods from BMJ Open Quality journal.BMJ Open Quality 2018;7:e000288. doi:10.1136/ bmjoq-2017-000288

Received 14 December 2017 Revised 17 April 2018 Accepted 4 May 2018

\section{Check for updates}

${ }^{1}$ Respiratory Medicine, Queen Elizabeth Hospital, Birmingham, UK

${ }^{2}$ Institute of Inflammation and Ageing, University of Birmingham, Birmingham, UK ${ }^{3}$ Rheumatology, University Hospitals Birmingham NHS Foundation Trust, Birmingham, UK

${ }^{4}$ Respiratory Medicine, Heart of England NHS Foundation Trust, Birmingham, UK

Correspondence to Dr Onn Shaun Thein; shaunthein@gmail.com

\section{ABSTRACT}

Oxygen is an important drug frequently used in the management of acutely unwell hospital patients. However, oxygen overuse can have fatal side effects particularly for those patients at risk of iatrogenic hypercapnia. British Thoracic Society Guidelines state that oxygen must be prescribed for all patients, with target saturations stipulated on the prescription for patient safety. A quality improvement project was undertaken with the aim to improve the oxygen prescription rate across the respiratory ward at a district general hospital, over a period of 3 months. Quality improvement methods were implemented based on data analysis at each stage, following discussion with senior doctors and specialist nurses, and after reviewing previous quality improvement projects published on BMJ Open Quality. The initial interventions of poster reminders and multidisciplinary team education failed to significantly improve the rates of oxygen prescription. Use of a targeted intervention where stickers were placed above oxygen taps significantly improved prescription rate from $20 \%$ in the non-targeted group to $60 \%$ in the targeted group. This was based on a BMJ Open Quality published improvement method. The current guidelines from the British Thoracic Society, and hospital's own guidelines, advise good oxygen prescribing. However, these recommendations alone are ineffective at achieving compliance among prescribers. Further targeted interventions have shown improvements in oxygen prescriptions and could lead to better clinical practice, patient care and safety.

\section{PROBLEM}

Oxygen is one of the most frequently prescribed inpatient drugs, but accurate prescription still poses a nationwide problem. ${ }^{1}$ The British Thoracic Society (BTS) 2015 audit showed $14 \%$ of hospital inpatients were on oxygen, with $42.5 \%$ of these not having a valid prescription. ${ }^{2}$ Patients who retain carbon dioxide due to underlying conditions, such as chronic obstructive pulmonary disease (COPD), bronchiectasis and some neuromuscular disorders, require a level of hypoxia to stimulate respiratory drive. Obliteration of that drive from iatrogenic overoxygenation can cause hypercapnia and its related complications. ${ }^{34}$
Both compliance with oxygen prescription and correct target saturations on the specialist respiratory ward at Solihull General Hospital was poor. Patients with exacerbations of COPD and type 2 (hypercapnic) respiratory failure were commonly admitted to this ward. It was therefore identified that potentially susceptible patients were at risk of serious complications of poor oxygen therapy.

The aim was to improve the oxygen prescription rate across the specialist respiratory ward in Solihull General Hospital to $\geq 80 \%$ in 6 weeks by reproducing intervention success from past BMJ Open Quality studies. ${ }^{5}$

\section{BACKGROUND}

BTS Guidelines state that oxygen must be prescribed for all patients on admission, with target saturations stipulated on the prescription. ${ }^{6}$ This would enable appropriate amounts of oxygen to be administered should acute hypoxaemia occur at any point during the patient's inpatient stay. Target saturations are often simplified into two ranges: $88 \%-92 \%$, usually for those at risk of hypercapnia, or 94\%-98\%. Various studies have provided evidence that administering high concentrations of oxygen to patients with severe exacerbations of COPD can increase the mortality risk. Poor oxygen prescription in UK clinical practice is a well-described problem, with over $40 \%$ of inpatient oxygen administration being delivered without an oxygen prescription. ${ }^{2}$ Lack of awareness has been addressed by educating prescribers and nurses, and previous oxygen-related quality improvement projects (QIPs) have documented improved compliance through use of education and other targeted interventions. ${ }^{5} 78$, These included, but were not limited to, stickers by oxygen taps and specific teaching at junior doctor training sessions.

These improvements have been carried out in the setting of paper prescription use. Electronic prescribing is implemented across 
the Heart of England NHS Foundation Trust, of which Solihull General Hospital is a part. This means oxygen prescription is standardised, leaving only route, frequency (continuous vs as required) and target saturations to prescriber discretion. Audit, therefore, is straightforward, and with simpler prescribing, we would expect high baseline prescription compliance.

\section{MEASUREMENT}

Baseline data were collected for all patients admitted to the respiratory ward over a single day (1 February 2017, $\mathrm{n}=27$ ). The electronic prescribing record, along with the patient notes and bedside charts were used. The data collected included if the patient was currently receiving oxygen, whether oxygen had been prescribed, whether a target range had been specified and whether there was a recorded diagnosis of a condition that could put the patient at risk of hypercapnic respiratory failure. From this, it was possible to determine the overall oxygen prescription rate and whether a safe prescription had been specified. A spreadsheet proforma was created in Microsoft Excel to record the data.

A percentage of 29.6 ( 8 of 27) of all patients were prescribed oxygen. Eight patients were actually receiving oxygen, $50 \%$ of which was prescribed. Ninety per cent of those deemed at risk of hypercapnia had a documented appropriate target saturation range. Patients deemed at risk of hypercapnia were those with a diagnosis of COPD, severe chest wall or spinal disease, neuromuscular disease, severe obesity, cystic fibrosis and bronchiectasis.

Interventions were then applied, and data were recollected after a minimum 1-week interval postintervention. Over the time period, three interventions were implemented.

\section{DESIGN}

The project team consisted of the four junior doctors working on the respiratory ward. Interventions were implemented based on data analysis at each stage, discussion with other members of the ward healthcare team including senior doctors and specialist nurses and from previous QIPs.

Initially, the quality improvement team discussed ideas and decided on which to implement first. The interventions were focused around increasing awareness of the issue on the ward. Data were recollected after 1 week following implementation of each intervention and were then analysed. Following the results, the next intervention was discussed among the team and was implemented.

At least one project team member was present on the ward each weekday, which ensured the interventions were implemented. Communication between the team occurred in person and electronically, and other staff members on the ward were consulted directly.

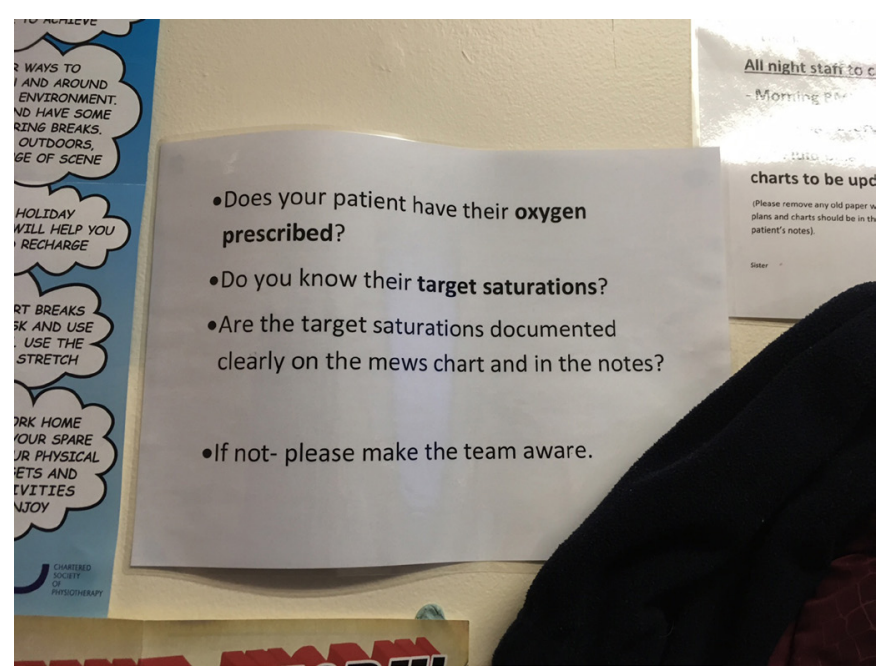

Figure 1 Oxygen sign used for the first intervention.

\section{STRATEGY}

We completed three plan, do, study and act (PDSA) cycles during the 6 -week period.

PDSA cycle 1 (1 January 2017-13 February 2017): the first intervention was to put up signs on the ward to increase staff awareness (figure 1). The signs prompted oxygen prescribing and target saturation documentation. The signs were placed both on the clinical ward areas and in the staff room area. Although a passive approach, the signs were intended to always be present so that multiple sets of shift working nurses and doctors could be exposed. We also directed senior nursing staff on the ward to alert rotating staff to these signs.

PDSA cycle 2 (1 March 2017-14 March 2017): our next intervention involved a more active approach. A member of the improvement team actively prompted members of the ward multidisciplinary team (MDT) at the regular morning board round meeting to check oxygen prescriptions and target saturations. This was done daily for 1 week by the junior doctor present and ensured that the nurse in charge for the day was alerted. We asked that the nurse in charge, and other members at the MDT meeting, relay the alert to other staff on shift.

PDSA cycle 3 (16 March 2017-1 April 2017): we had tried our own change ideas and interventions aimed at the ward staff as a whole. Our next step was to look for change methods that had been effective elsewhere. With further research into previous QIPs, we discovered a targeted intervention implemented by Helliar. ${ }^{5}$ in Southampton, which demonstrated improved prescription compliance. This suggested that targeting staff who were actively administering oxygen was more effective than repeated education events. Drawing on their reminder sign, we developed a sign (figure 2 ) to be placed directly next to the oxygen taps to remind nursing staff to ask doctors to prescribe oxygen for patients. Design and placement were in conjunction with ward nursing staff to increase awareness and to develop effective signs. The signs were used in each bed space in a single bay on the ward. 


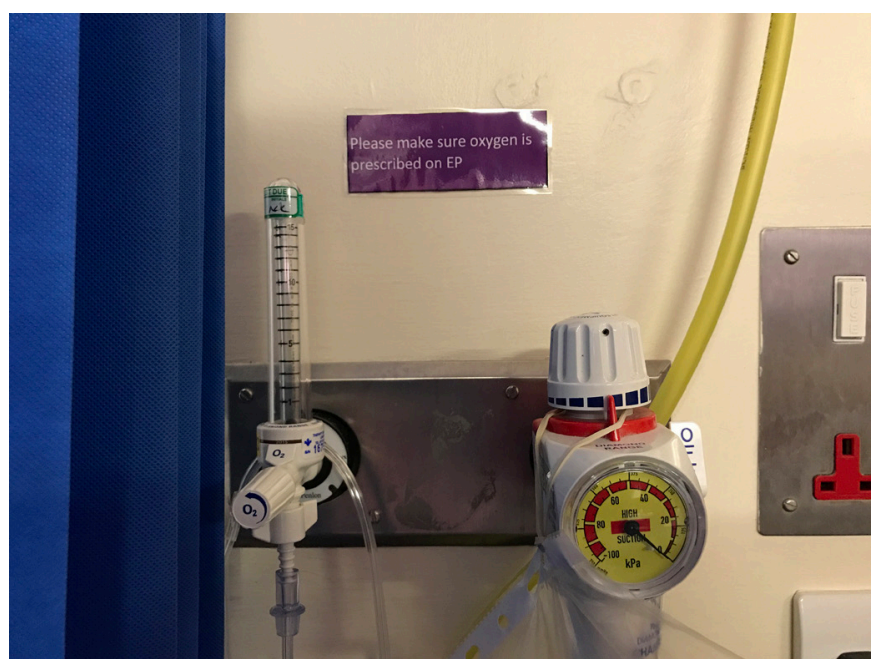

Figure 2 Oxygen sign used for the third intervention.

\section{RESULTS}

Each round of data collection was carried out on a single day, at intervals over the 6 weeks. All of the patients on the ward at the time of collection were intended to be included. The maximum ward capacity was 27 patients, although there were occasionally unoccupied beds.

\section{PDSA cycle 1}

The oxygen prescription rate did not improve (18.5\%), but lower target saturations were prescribed for those who should have $(100 \%)$. We found that this intervention was not reliable, as the signs fell down, were not noticeable among the various other posters also present or were obstructed from view. Therefore, awareness was not increased.

\section{PDSA cycle 2}

There was improvement from the previous cycle as the oxygen prescription rate increased to $27 \%$, but this was not an improvement from baseline. This strategy relied on individuals to pass on information, which may not have always occurred. Moreover, a member of the improvement team needed to be present at the meeting, otherwise the announcement would not be made, and so this method would not be sustainable.

\section{PDSA cycle 3}

There was no improvement in our main outcome of oxygen prescription rate on the respiratory ward. The rate at baseline, at the beginning of February, was 29.6\%, compared with $30.0 \%$ after the third cycle in mid-March as shown in figure 3. However, there was a significant improvement in oxygen prescription rate when the targeted signs in PDSA 3 were used in the single bay. Sixty per cent of patients in this targeted bay were prescribed oxygen compared with $20 \%$ of the patients on the rest of the ward as shown in figure 4 .

\section{LESSONS AND LIMITATIONS}

For an intervention to be sustainable and effective beyond just the short term, ongoing active involvement from all members of a team is vital. The project members were all rotating junior doctors, who were working on the ward for 4 months only. Permanent staff members, including consultants and nurses, were consulted but were not core project team members. As learnt during cycle 3, ward staff should be involved in the design and implementation of interventions to improve efficacy and sustainability. For

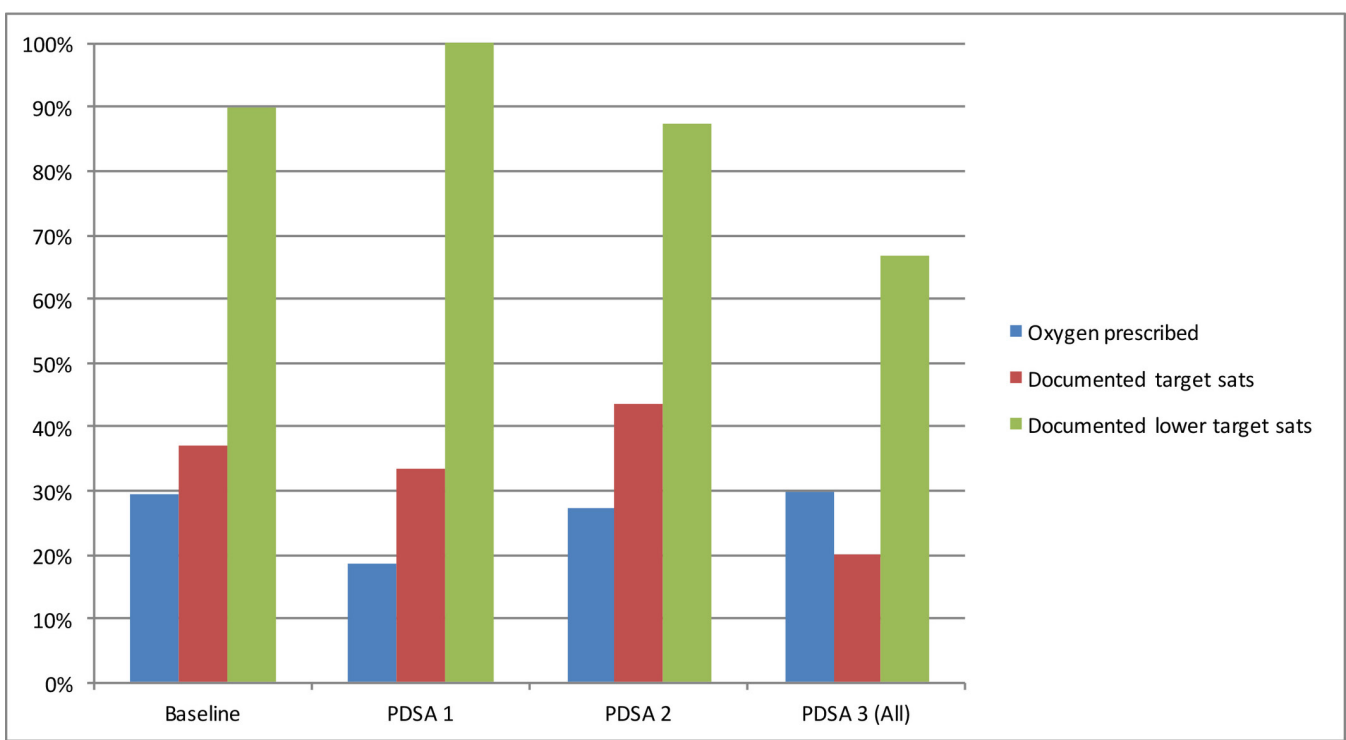

Figure 3 Percentage of patients with oxygen prescriptions and documented target saturations at subsequent PDSA cycles. Blue bars represent percentage of patients with oxygen prescribed. Red bars represent patients with their target saturations documented either on electronic prescribing or the observation chart. Green bars represent patients at risk of iatrogenic hypercapnia with target saturations documented either on electronic prescribing or the observation chart. (PDSA= plan, do, study and act). 


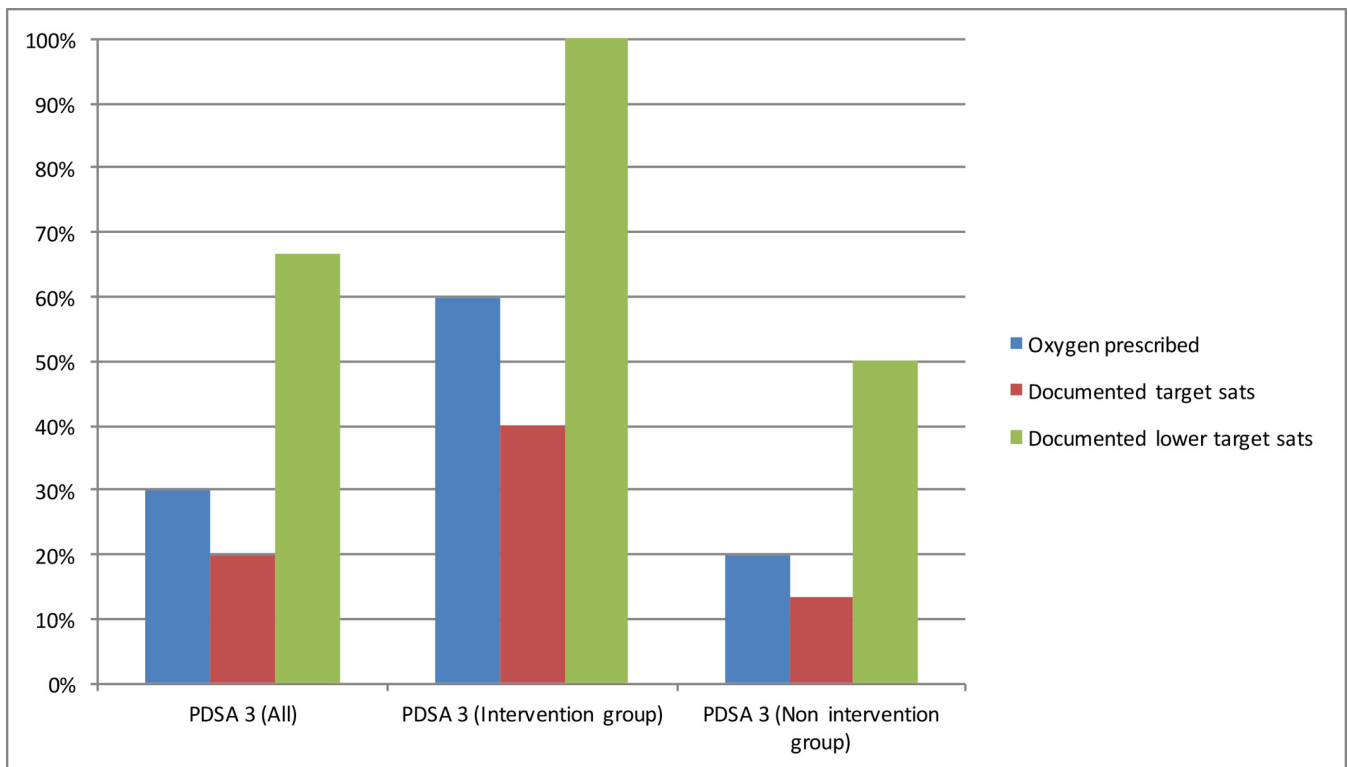

Figure 4 PDSA cycle 3 breakdown into intervention and non-intervention groups. Percentage of patients with oxygen prescriptions and documented target saturations. Blue bars represent percentage of patients with oxygen prescribed. Red bars represent patients with their target saturations documented either on electronic prescribing or the observation chart. Green bars represent patients at risk of iatrogenic hypercapnia with target saturations documented either on electronic prescribing or the observation chart.

instance, the signs in cycle 1 were less noticeable than the signs in cycle 3 .

Despite improved compliance, interventions were targeted to a single bay in PDSA cycle 3 due to the cost needed to create individual signs for each oxygen tap and time constraints of the QIP. This was an unexpected expense associated with the third intervention and not previously documented. We specifically targeted a high dependency bay where compliance with oxygen would be most crucial to patient care.

Frequent communication and coordination are important in the process of a QIP. ${ }^{9}$ At the beginning of the project, the team lacked specific leadership and designation. For instance, it was not clear who was going to design, print and put up the signs, and the signs used did not undergo a team review or approval process. Inconsistency in team member presence, for example, due to on-call commitments, was also not considered. Planned, regular team meetings would help to keep PDSA cycles and a QIP on track.

Our project had limitations. First, the core project team members were four of the respiratory ward junior doctors, although there were other junior doctors regularly on the ward and in the admissions unit. It is possible that being directly involved in the QIP itself was an intervention, leading to the project members increasing their oxygen prescribing. The identity of prescribers was not collected to assess this. The high turnover nature of the ward and the nature of the QIP would mean that new admissions to the ward would not have been affected by our intervention. In addition to this, they would not have had a review from the ward team to update their prescription. Disseminating the education and results of the QIP beyond the respiratory ward would be beneficial to improve prescription compliance. All acute medical patients are admitted through the acute medical unit, and so implementing interventions here would be beneficial to increase compliance 'at the front door' and, as a result, throughout the other hospital wards.

The number of ward patients included in each cycle of data collection should be considered. The maximum capacity of the ward is 27 , but in some of the cycles, the sample size was fewer than this. Moreover, the targeted intervention in PDSA cycle 3 was implemented on a very small sample of five patients in a single bay. Other potential confounding factors may have been the specific nurse or doctor working in that specific bay. The intervention should be more widely implemented and over a longer period of time to assess whether the intervention is more universally effective.

\section{CONCLUSION}

Oxygen prescription remains complex for a drug that is administered so frequently. The aim of this QIP was to increase the oxygen prescription rate on the respiratory ward at Solihull General Hospital to at least $80 \%$. We did not achieve this target, but we identified a potential sustainable intervention, which could help to achieve this in the future. Our findings suggest that using a focused, targeted intervention can effectively improve compliance with oxygen prescriptions. This positive outcome will need to be implemented further throughout the respiratory ward and further to the other hospital wards and trust-wide. 
This is consistent with other oxygen prescription audits that have demonstrated improvement after targeted visual intervention. ${ }^{578}$ Although efficacy has been previously documented, it still remains absent from most hospitals. Our setting differed from previous models that centred around paper prescriptions. The use of electronic prescribing does not intrinsically improve prescribing as shown from our results. Further testing and proving reproducibility will hope to show this is a simple and affordable but effective and sustainable method to improve patient safety.

Acknowledgements The authors would like to thank the contributions of the nursing staff on Ward 19 at Solihull General Hospital and in particular Respiratory Specialist Nurse Katie Neighbour.

Contributors OST, CMTC, EM and MM designed the project and collection tool and collected data. OST analysed the data. OST and CMTC drafted and edited the manuscript. DD reviewed manuscript.

Funding The authors have not declared a specific grant for this research from any funding agency in the public, commercial or not-for-profit sectors.

Competing interests None declared.

Provenance and peer review Not commissioned; externally peer reviewed.

Open Access This is an Open Access article distributed in accordance with the Creative Commons Attribution Non Commercial (CC BY-NC 4.0) license, which permits others to distribute, remix, adapt, build upon this work non-commercially, and license their derivative works on different terms, provided the original work is properly cited and the use is non-commercial. See: http://creativecommons.org/ licenses/by-nc/4.0/
C Published by the BMJ Publishing Group Limited. For permission to use (where not already granted under a licence) please go to http://www.bmj.com/company/ products-services/rights-and-licensing/

\section{REFERENCES}

1. Dickson C. Oxygen Delivery on Medical Wards. BMJ Qual Improv Rep 2015;4:u206934.w2785.

2. O'Driscoll R. British Thoracic Society Emergency oxygen audit report. BTS National Respiratory Audit Programme. Annual Report. 2015 https://www.brit-thoracic.org.uk/document-library/audit-and-qualityimprovement/audit-reports/bts-emergency-oxygen-audit-report-2015/ (accessed 3 Oct 2017).

3. Murphy R, Driscoll P, O'Driscoll R. Emergency oxygen therapy for the COPD patient. Emerg Med J 2001;18:333-9.

4. Plant PK, Owen JL, Elliott MW. One year period prevalence study of respiratory acidosis in acute exacerbations of COPD: implications for the provision of non-invasive ventilation and oxygen administration. Thorax 2000;55:550-4.

5. Helliar S. Improving oxygen prescribing rates by tailoring interventions for specific healthcare professional groups. BMJ Qual Improv Rep 2016;5:u209520.w4033.

6. O'Driscoll BR, Howard LS, Earis J, et al. BTS guideline for emergency oxygen use in adult patients. Thorax 2017;72(Suppl 1:ii1-ii90.

7. Gatter M, Dixon G, Wall J, et al. Changing an ingrained culture: Improving the safety of oxygen therapy at University Hospitals Bristol NHS Foundation Trust. BMJ Qual Improv Rep 2015;4:u203238.w1474

8. Dimock RAC, Stuart S, Padmanaban V, et al. P64 The impact of simple interventions on oxygen prescribing and monitoring: Audit of oxygen management in Central London Teaching Hospital. Thorax 2013:68:A103.2-A104.

9. American Association of Family Physicians. Basics of Quality Improvement. https://www.aafp.org/practice-management/ improvement/basics.html (accessed 14 Dec 2017). 